\title{
Equitable aggregations and multiple criteria analysis
}

\author{
Michael M. Kostreva ${ }^{a}$, Włodzimierz Ogryczak ${ }^{\text {b,* }}$, Adam Wierzbicki ${ }^{\text {c }}$ \\ a Department of Mathematical Sciences, Clemson University, Clemson, SC 29634, USA \\ ${ }^{\mathrm{b}}$ Institute of Control and Computation Engineering, Warsaw University of Technology, Nowowiejska 15/19, 00-665 Warsaw, Poland \\ ${ }^{\mathrm{c}}$ Polish-Japanese Institute of Information Technology, Koszykowa 86, 02-008 Warsaw, Poland
}

Received 1 July 2002; accepted 1 June 2003

Available online 8 October 2003

\begin{abstract}
In the past decade, increasing interest in equity issues resulted in new methodologies in the area of operations research. This paper deals with the concept of equitably efficient solutions to multiple criteria optimization problems. Multiple criteria optimization usually starts with an assumption that the criteria are incomparable. However, many applications arise from situations which present equitable criteria. Moreover, some aggregations of criteria are often applied to select efficient solutions in multiple criteria analysis. The latter enforces comparability of criteria (possibly rescaled). This paper presents aggregations which can be used to derive equitably efficient solutions to both linear and nonlinear multiple optimization problems. An example with equitable solutions to a capital budgeting problem is analyzed in detail. An equitable form of the reference point method is introduced and analyzed.
\end{abstract}

(c) 2003 Elsevier B.V. All rights reserved.

Keywords: Multiple criteria; Efficiency; Equity; Fairness; Ordered weighted averaging; Lexicographic minimax; Reference point method

\section{Introduction}

The problem of multiple criteria optimization has been studied for many years, and the techniques of multiple criteria analysis have found success in many diverse applications $[23,24]$. The standard approach starts with an assumption that the criteria are incomparable, i.e. having no basis of comparison. However, there are many applications in which the criteria are uniform in the sense

\footnotetext{
${ }^{*}$ Corresponding author. Tel.: +48-22-658-3165; fax: +48-22658-3164.

E-mail address: ogryczak@ia.pw.edu.pl (W. Ogryczak).
}

of the scale used and their values are directly comparable. Moreover, the criteria are considered impartially which makes the distribution of outcomes more important than the assignment of several outcomes to the specific criteria. Such models express ideas of allocation of resources and try to achieve some equitable allocation of resources [10]. More generally, the models are related to the evaluation of various systems which serve many users where quality of service for every individual user defines the criteria. An example arises in location theory, in which the clients of a system are entitled to equitable treatment according to community regulations. In such problems, the decisions often concern the placement of a service 
center or other facility in a position so that the users are treated in an equitable way, relative to certain criteria [16]. Another type of model is that of approximation of discrete data by a functional form. The residuals may be viewed as objectives to be minimized, and in the classical approach, there is no reason to treat them in any way but equitably. Moreover, uniform individual objectives may be associated with some events rather than physical users, like in many dynamic optimization problems where uniform individual criteria represent a similar event in various periods.

Recently, one may notice an increasing interest in equity (or fairness) issues in the area of Operations Research. Several research publications dealing with the issue with respect to various application areas have appeared [4,10,12,16,17]. Some of them directly related equity to the multiple criteria optimization methodology. Finally, the novel and distinct mathematical approach denoted by equitable efficiency has been developed to provide solutions to these examples of multiple criteria optimization. The formalization of the equitable efficiency was introduced in our earlier paper [6], which analyzes the basic solution properties and the basic generation techniques. This has already allowed the solution of problems arising in location theory $[7,16]$ as well as in portfolio optimization [17]. Further progress made in generation techniques [20] extends possible areas of application of the equitable multiple criteria optimization.

The concept of equitably efficient solution is a specific refinement of the Pareto-optimality. Hence, equitable multiple criteria techniques focus on some selection of Pareto-optimal solutions. It turns out, however, that the techniques are often applied to select efficient solutions in general multiple criteria optimization. Indeed, in approaches which seek to scalarize the multiple criteria, some effort is always placed to replace the original objective functions with some individual achievements which are combined to form a final scalar objective function to be optimized. This is done in order to make the physical units of the individual achievements uniform, so that they can be added or otherwise composed. This phase of the modeling discipline seldom questions the process or the consequences of such a uniformization. One of the results of our research is to trace the consequences of this uniformization beyond the process of aggregation of functions for scalarization. We will show that every efficient solution of a multiple criteria optimization problem can be identified by the optimization of an equitable aggregation applied to appropriately defined individual achievements.

The paper is organized as follows. In the next section we recall and explain in detail the concept of equitable dominance and equitably efficient solutions to multiple criteria optimization problems. Section 3 is devoted to equitable aggregations. It is shown that while various $L_{p}$ norms can be used as equitable aggregations for positive outcomes, the ordered weighted aggregations are applicable for general (positive or negative) outcomes. Further, in Section 4 we examine various applications of equitable optimization to multiple criteria analysis. First we show that several multiple criteria problems require, in fact, equitable preferences models and equitable aggregation may result in an efficient solution procedure. Next, we analyze equitable approaches to general multiple criteria problems which by introducing individual achievements are transformed into uniform and equitable problems. The latter covers in particular the reference point and goal programming methodology.

\section{Pareto-optimality and equitable efficiency}

Consider a decision problem defined as an optimization problem with $m$ objective functions $f_{i}(\mathbf{x})$. For simplification we assume, without loss of generality, that the objective functions are to be minimized. The problem can be formulated as follows:

$\min \{\mathbf{f}(\mathbf{x}): \mathbf{x} \in Q\}$,

where $\mathbf{f}(\mathbf{x})$ is a vector-function that maps the decision space $X=R^{n}$ into the criterion space $Y=R^{m}, Q \subset X$ denotes the feasible set, and $\mathbf{x} \in X$ denotes the vector of decision variables.

We refer to the elements of the criterion space as outcome vectors. 
Model (1) only specifies that we are interested in minimization of all objective functions $f_{i}$ for $i \in I=\{1,2, \ldots, m\}$. In order to make it operational, one needs to assume some solution concept specifying what it means to minimize multiple objective functions. The solution concepts are defined by properties of the corresponding preference model. We assume that solution concepts depend only on evaluation of the outcome vectors while not taking into account any other solution properties not represented within the outcome vectors. Thus, we can limit our considerations to the preference model in the criterion space $Y$.

The preference model is completely characterized by the relation of weak preference [9], denoted hereafter with $\preceq$. Namely, the corresponding relations of strict preference $\prec$ and indifference $\cong$ are defined by the following formulas:

$\mathbf{y}^{\prime} \prec \mathbf{y}^{\prime \prime} \Longleftrightarrow\left(\mathbf{y}^{\prime} \preceq \mathbf{y}^{\prime \prime}\right.$ and not $\left.\mathbf{y}^{\prime \prime} \preceq \mathbf{y}^{\prime}\right)$,

$\mathbf{y}^{\prime} \cong \mathbf{y}^{\prime \prime} \Longleftrightarrow\left(\mathbf{y}^{\prime} \preceq \mathbf{y}^{\prime \prime}\right.$ and $\left.\mathbf{y}^{\prime \prime} \preceq \mathbf{y}^{\prime}\right)$.

The preference model related to the standard Pareto-optimal solution concept also assumes that the preference relation $\preceq$ is reflexive,

$\mathbf{y} \preceq \mathbf{y}$,

transitive,

$\left(\mathbf{y}^{\prime} \preceq \mathbf{y}^{\prime \prime}\right.$ and $\left.\mathbf{y}^{\prime \prime} \preceq \mathbf{y}^{\prime \prime \prime}\right) \Rightarrow \mathbf{y}^{\prime} \preceq \mathbf{y}^{\prime \prime \prime}$,

and strictly monotonic,

$\mathbf{y}-\varepsilon \mathbf{e}_{i} \prec \mathbf{y} \quad$ for $\varepsilon>0, i \in I$,

where $\mathbf{e}_{i}$ denotes the $i$ th unit vector in the criterion space. The last assumption expresses the fact that for each individual objective function less is better (minimization). The preference relations satisfying axioms (4)-(6) are called hereafter rational preference relations. The rational preference relations allow us to formalize the Pareto-optimal solution concept with the following definitions. We say that outcome vector $\mathbf{y}^{\prime} \in Y$ rationally dominates $\mathbf{y}^{\prime \prime} \in Y$ $\left(\mathbf{y}^{\prime} \prec_{r} \mathbf{y}^{\prime \prime}\right)$, iff $\mathbf{y}^{\prime} \prec \mathbf{y}^{\prime \prime}$ for all rational preference relations $\preceq$. We say that a feasible solution $\mathbf{x} \in Q$ is a Pareto-optimal (or efficient) solution of the multiple criteria problem (1), iff $\mathbf{y}=\mathbf{f}(\mathbf{x})$ is rationally nondominated.
The relation of weak rational dominance $\preceq_{r}$ may be expressed in terms of the vector inequality: $\mathbf{y}^{\prime} \preceq_{r} \mathbf{y}^{\prime \prime}$ iff $y_{i}^{\prime} \leqslant y_{i}^{\prime \prime}$ for all $i \in I$. As a consequence, we can state that a feasible solution $\mathbf{x}^{0} \in Q$ is a Pareto-optimal solution of the multiple criteria problem (1), if and only if, there does not exist $\mathbf{x} \in Q$ such that $f_{i}(\mathbf{x}) \leqslant f_{i}\left(\mathbf{x}^{0}\right)$ for all $i \in I$ where at least one strict inequality holds. The latter refers to the commonly used definition of the Pareto-optimal solutions as feasible solutions for which one cannot improve any criterion without worsening another [23]. However, the axiomatic definition of the rational preference relation allows us to introduce additional properties of the preferences related to the uniform and equitable outcomes.

While dealing with uniform criteria, we want to focus on the distribution of outcome values while ignoring their ordering. That means, in the multiple criteria optimization problem (1) we are interested in a set of values of the criteria without taking into account which criterion is taking a specific value. In other words, a solution generating individual outcomes: 4,2 and 0 for criteria $f_{1}$, $f_{2}$ and $f_{3}$, respectively, should be considered equally good as a solution generating outcomes 0 , 2 and 4 . Hence, we assume that the preference model is impartial (anonymous, symmetric). In terms of the preference relation it may be written as the following axiom:

$\left(y_{\tau(1)}, y_{\tau(2)}, \ldots, y_{\tau(m)}\right) \cong\left(y_{1}, y_{2}, \ldots, y_{m}\right)$

for any permutation $\tau$ of $I$. Further, according to the theory of equity measurement [10], the preference model should satisfy the (Pigou-Dalton) principle of transfers. The principle of transfers states that a transfer of any small amount from an outcome to any other relatively worse-off outcome results in a more preferred outcome vector. As a property of the preference relation, the principle of transfers takes the form of the following axiom:

$y_{i^{\prime}}>y_{i^{\prime \prime}} \Rightarrow \mathbf{y}-\varepsilon \mathbf{e}_{i^{\prime}}+\varepsilon \mathbf{e}_{i^{\prime \prime}} \prec \mathbf{y} \quad$ for $0<\varepsilon<y_{i^{\prime}}-y_{i^{\prime \prime}}$.

Thus a solution generating all three outcomes equal to 2 is considered better than any solution generating individual outcomes: 4,2 and 0 . The preference relations satisfying all axioms (4)-(8) we will call hereafter equitable rational preference relations. 
Requirements of impartiality (7) and the principle of transfers (8) do not contradict the multiple criteria optimization axioms (4)-(6). Therefore, we can consider equitable multiple criteria optimization [6] based on the preference model defined by axioms (4)-(8). The equitable rational preference relations allow us to define the concept of equitably efficient solution, similar to the standard efficient (Pareto-optimal) solution defined with the rational preference relations. We say that outcome vector $\mathbf{y}^{\prime}$ equitably dominates $\mathbf{y}^{\prime \prime}\left(\mathbf{y}^{\prime} \prec_{e} \mathbf{y}^{\prime \prime}\right)$, iff $\mathbf{y}^{\prime} \prec \mathbf{y}^{\prime \prime}$ for all equitable rational preference relations $\preceq$. We say that a feasible solution $\mathbf{x} \in Q$ is equitably efficient (is an equitably efficient solution of the multiple criteria problem (1), if and only if there does not exist any $\mathbf{x}^{\prime} \in Q$ such that $\mathbf{f}\left(\mathbf{x}^{\prime}\right) \prec_{e} \mathbf{f}(\mathbf{x})$. Note that each equitably efficient solution is also a Pareto-optimal solution, but not vice verse. For instance, having two possible solutions generating outcome vectors $\mathbf{y}^{\prime}=(5,0,5)$ and $\mathbf{y}^{\prime \prime}=(0,3,0)$, respectively, we recognize both the solutions as Pareto-optimal. In fact, neither $\mathbf{y}^{\prime}$ dominates rationally $\mathbf{y}^{\prime \prime}$ nor $\mathbf{y}^{\prime \prime}$ dominates $\mathbf{y}^{\prime}$. However, the first solution generates two outcomes equal to 5 and one outcome equal to 0 , whereas the second solution generates one outcome equal to 3 and two outcomes equal to 0 . Thus, the second outcome vector is clearly better in terms of distribution of outcomes and $\mathbf{y}^{\prime \prime}$ equitably dominates $\mathbf{y}^{\prime}$.

The relation of equitable dominance $\preceq_{e}$ can be expressed as a vector inequality on the cumulative ordered outcomes. This can be mathematically formalized as follows. First, introduce the ordering map $\Theta: R^{m} \rightarrow R^{m}$ such that $\Theta(\mathbf{y})=\left(\theta_{1}(\mathbf{y}), \theta_{2}(\mathbf{y})\right.$, $\left.\ldots, \theta_{m}(\mathbf{y})\right), \quad$ where $\theta_{1}(\mathbf{y}) \geqslant \theta_{2}(\mathbf{y}) \geqslant \cdots \geqslant \theta_{m}(\mathbf{y})$ and there exists a permutation $\tau$ of set $I$ such that $\theta_{i}(\mathbf{y})=y_{\tau(i)}$ for $i=1,2, \ldots, m$. Next, apply to ordered outcomes $\Theta(\mathbf{y})$, a linear cumulative map thus resulting in the cumulative ordering map $\bar{\Theta}(\mathbf{y})=\left(\bar{\theta}_{1}(\mathbf{y}), \bar{\theta}_{2}(\mathbf{y}), \ldots, \bar{\theta}_{m}(\mathbf{y})\right)$ defined as

$\bar{\theta}_{i}(\mathbf{y})=\sum_{j=1}^{i} \theta_{j}(\mathbf{y}) \quad$ for $i=1, \ldots, m$.

The coefficients of vector $\bar{\Theta}(\mathbf{y})$ express, respectively: the largest outcome, the total of the two largest outcomes, the total of the three largest outcomes, etc.
The relation $\bar{\Theta}\left(\mathbf{y}^{\prime}\right) \leqslant \bar{\Theta}\left(\mathbf{y}^{\prime \prime}\right)$ was extensively analyzed within the theory of majorization [13], where it is called the relation of weak submajorization. The theory of majorization includes the results which allow us to derive the following theorem [6].

Theorem 1. Outcome vector $\mathbf{y}^{\prime} \in Y$ equitably dominates $\mathbf{y}^{\prime \prime} \in Y$, if and only if $\bar{\theta}_{i}\left(\mathbf{y}^{\prime}\right) \leqslant \bar{\theta}_{i}\left(\mathbf{y}^{\prime \prime}\right)$ for all $i \in I$ where at least one strict inequality holds.

Vector $\bar{\Theta}(\mathbf{y})$ can be viewed graphically with a piecewise linear curve connecting point $(0,0)$ and points $\left(i / m, \bar{\theta}_{i}(\mathbf{y}) / m\right)$ for $i=1, \ldots, m$. Such a curve represents the (upper) absolute Lorenz curve which can be mathematically formalized as follows. First, we introduce the left-continuous right tail cumulative distribution function

$F_{\mathbf{y}}(d)=\sum_{i=1}^{m} \frac{1}{m} \delta_{i}(d) \quad$ where

$\delta_{i}(d)= \begin{cases}1 & \text { if } y_{i} \geqslant d, \\ 0 & \text { otherwise }\end{cases}$

which for any real value $d$ provides the measure of outcomes greater or equal to $d$. Next, we introduce the quantile function $F_{\mathbf{y}}^{(-1)}$ as the right-continuous inverse of the cumulative distribution function $F_{\mathrm{y}}$ : $F_{\mathbf{y}}^{(-1)}(\eta)=\sup \left\{d: F_{\mathbf{y}}(d) \geqslant \eta\right\}$ for $0<\eta \leqslant 1$.

By integrating $F_{\mathbf{y}}^{(-1)}$ one gets

$$
\begin{aligned}
F_{\mathbf{y}}^{(-2)}(0) & =0 \text { and } F_{\mathbf{y}}^{(-2)}(\eta) \\
& =\int_{0}^{\eta} F_{\mathbf{y}}^{(-1)}(\alpha) \mathrm{d} \alpha \quad \text { for } 0<\eta \leqslant 1 .
\end{aligned}
$$

Graphs of functions $F_{\mathbf{y}}^{(-2)}(\eta)$ (with respect to $\eta$ ) take the form of concave curves (Fig. 1), the (upper) absolute Lorenz curves. In our case of $m$ outcomes, the absolute Lorenz curve is completely defined by the values $F_{\mathbf{y}}^{(-2)}(i / m)=\frac{1}{m} \bar{\theta}_{i}(\mathbf{y})$ for $i=$ $1, \ldots, m$ where $F_{\mathbf{y}}^{(-2)}(1 / m)=\bar{\theta}_{1}(\mathbf{y})=\theta_{1}(\mathbf{y})$ represent the worst outcome and $F_{\mathbf{y}}^{(-2)}(1)=$ $\frac{1}{m} \bar{\theta}_{m}(\mathbf{y})=\frac{1}{m} \sum_{i=1}^{m} \theta_{i}(\mathbf{y})$.

In income economics the Lorenz curve is a cumulative population versus income curve [13]. A perfectly equal distribution of income has the diagonal line as the Lorenz curve and no outcome 


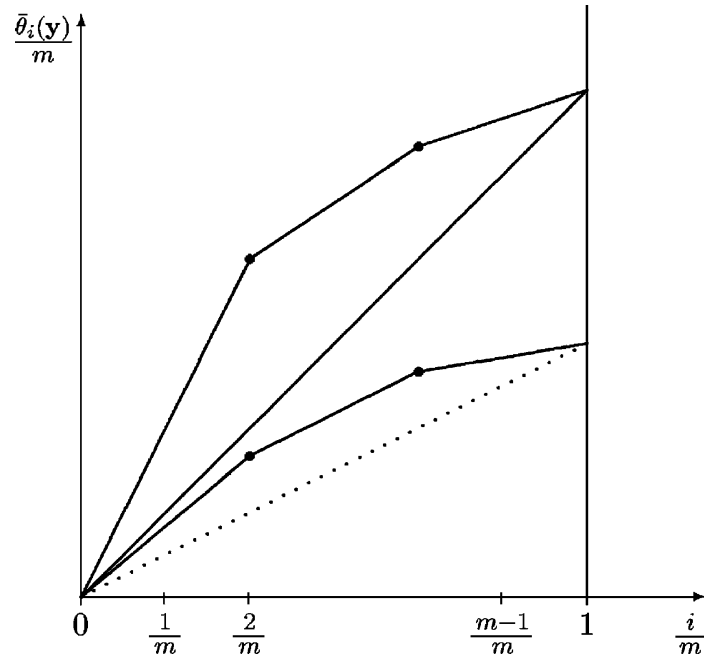

Fig. 1. $\bar{\Theta}(\mathbf{y})$ as the upper absolute Lorenz curves.

vector can be better. The absolute Lorenz curves, used in the equitable optimization, are unnormalized taking into account also values of outcomes. Vectors of equal outcomes are distinguished according to the value of outcomes. They are graphically represented with various ascent lines in Fig. 1. Hence, with the relation of equitable dominance an outcome vector of small unequal outcomes may be preferred to an outcome vector with large equal outcomes. This allows to overcome the common flaws of the approaches based on a strict inequality minimization.

Note that Theorem 1 permits one to express equitable efficiency for problem (1) in terms of the Pareto-optimality for the multiple criteria problem with objectives $\bar{\Theta}(\mathbf{f}(\mathbf{x}))$ :

$\min \left\{\left(\bar{\theta}_{1}(\mathbf{f}(\mathbf{x})), \bar{\theta}_{2}(\mathbf{f}(\mathbf{x})), \ldots, \bar{\theta}_{m}(\mathbf{f}(\mathbf{x}))\right): \mathbf{x} \in Q\right\}$.

Corollary 1. A feasible solution $\mathbf{x} \in Q$ is an equitably efficient solution of the multiple criteria problem (1), iff it is a Pareto-optimal solution of the multiple criteria problem (12).

Corollary 1 provides the relationship between equitable efficiency and Pareto-optimality. Moreover, the multiple criteria problem (12) may serve as a source of techniques generating equitable efficient solutions to the original problem (1). Some equitable location models have taken advantages of this opportunity $[5,7,15,16]$. Although the definition of quantities $\bar{\theta}_{k}(\mathbf{y})$, used as criteria in (12), are very complicated they can be modeled with simple auxiliary variables and constraints. It is commonly known that the worst (largest) outcome may be defined by the following optimization: $\bar{\theta}_{1}(\mathbf{y})=\min \left\{t: t \geqslant y_{i}\right.$ for $\left.i=1, \ldots, m\right\}$, where $t$ is an unrestricted variable. It turns out that this approach can be generalized to provide an effective modeling technique for quantities $\bar{\theta}_{k}(\mathbf{y})$ with arbitrary $k$ [21]. Namely, for a given outcome vector $\mathbf{y}$ the quantity $\bar{\theta}_{k}(\mathbf{y})$ may be found by solving the following linear program:

$$
\begin{gathered}
\bar{\theta}_{k}(\mathbf{y})=\min \left\{k t+\sum_{i=1}^{m} d_{i}^{+}: t+d_{i}^{+} \geqslant y_{i}, d_{i}^{+} \geqslant 0\right. \text { for } \\
=1, \ldots, m\},
\end{gathered}
$$

where $t$ is an unrestricted variable while nonnegative variables $d_{i}^{+}$represent, for several outcome values $y_{i}$, their upside deviations from the value of $t$. Independently from the formal proof [21], this formula can be justified as follows. It is obvious that $\min \left(k t+\sum_{i=1}^{m} d_{i}^{+}\right)=\bar{\theta}_{k}(\mathbf{y})$ whenever no more than $k-1$ deviations $d_{i}^{+}$are strictly positive. On the other hand, for any $t$ and $d_{i}^{+}$feasible to (13) one can define an alternative feasible values: $\tilde{t}=t+\Delta$ and $\tilde{d}_{i}^{+}=d_{i}^{+}-\Delta$ for $d_{i}^{+}>0$, where $\Delta$ is an arbitrary small positive number. For at least $k$ positive values one gets $k \tilde{t}+\sum_{i=1}^{m} \tilde{d}_{i}^{+} \leqslant k t+$ $\sum_{i=1}^{m} d_{i}^{+}$, which justifies (13).

Formula (13) allows us to formulate problem (12) as the following multiple criteria optimization problem:

$\min \left(z_{1}, z_{2}, \ldots, z_{m}\right)$

subject to $\mathbf{x} \in Q$,

$z_{k}=k t_{k}+\sum_{i=1}^{m} d_{i k}^{+} \quad$ for $k=1, \ldots, m$,
$t_{k}+d_{i k}^{+} \geqslant f_{i}(\mathbf{x}), d_{i k}^{+} \geqslant 0 \quad$ for $i, k=1, \ldots, m$.

Note that problem (14)-(16) belongs to the class of convex programs provided that the feasible set $Q$ is 
convex and all the original criteria $f_{i}$ are convex functions. In the case of a linear multiple criteria problem (1) the resulting formulation (14)-(16) remains in the class of linear programs.

\section{Equitable aggregations}

Typical solution concepts for multiple criteria problems are defined by aggregation functions $g: Y \rightarrow R$ to be minimized. Thus the multiple criteria problem (1) is replaced with the minimization problem

$\min \{g(\mathbf{f}(\mathbf{x})): \mathbf{x} \in Q\}$.

In order to guarantee the consistency of the aggregated problem (17) with minimization of all individual objective functions in the original multiple criteria problem, the aggregation function must be strictly increasing with respect to every coordinate, i.e.

$$
\begin{aligned}
y_{i}^{\prime}<y_{i} \Rightarrow & g\left(y_{1}, \ldots, y_{i-1}, y_{i}^{\prime}, y_{i+1}, \ldots, y_{m}\right) \\
& <g\left(y_{1}, y_{2}, \ldots, y_{m}\right) \quad \text { for } i \in I .
\end{aligned}
$$

Every optimal solution to the aggregated problem (17) is then a Pareto-optimal solution of the original multiple criteria problem.

The aggregated problem (17) and its corresponding preference model are defined by the relation: $\mathbf{y}^{\prime} \preceq \mathbf{y}^{\prime \prime}$ iff $g\left(\mathbf{y}^{\prime}\right) \leqslant g\left(\mathbf{y}^{\prime \prime}\right)$. In order to guarantee equitable rationality of this preference relation, the aggregation function must be strictly increasing and symmetric (impartial),

$$
\begin{aligned}
& g\left(y_{\tau(1)}, y_{\tau(2)}, \ldots, y_{\tau(m)}\right)=g\left(y_{1}, y_{2}, \ldots, y_{m}\right) \\
& \quad \text { for any permutation } \tau \text { of } I,
\end{aligned}
$$

as well as equitable (to satisfy the principle of transfers),

$$
\begin{aligned}
& g\left(y_{1}, \ldots, y_{i^{\prime}}-\varepsilon, \ldots, y_{i^{\prime \prime}}+\varepsilon, \ldots, y_{m}\right) \\
& \quad<g\left(y_{1}, y_{2}, \ldots, y_{m}\right) \text { for } 0<\varepsilon<y_{i^{\prime}}-y_{i^{\prime \prime}} .
\end{aligned}
$$

In the case of an aggregation function satisfying all the requirements (18)-(20), we call the corresponding problem (17) an equitable aggregation of problem (1). Every optimal solution to the equitable aggregation (17) is an equitably efficient solution of the original multiple criteria problem.
Note that symmetric functions satisfying the requirement

$$
\begin{aligned}
& g\left(y_{1}, \ldots, y_{i^{\prime}}-\varepsilon, \ldots, y_{i^{\prime \prime}}+\varepsilon, \ldots, y_{m}\right) \\
& \quad \leqslant g\left(y_{1}, y_{2}, \ldots, y_{m}\right) \text { for } 0<\varepsilon<y_{i^{\prime}}-y_{i^{\prime \prime}}
\end{aligned}
$$

are called (weakly) Schur-convex [13] while the stronger requirement of equitability (20), we consider, is related to strictly Schur-convex functions. In other words, an aggregation (17) is equitable if it is defined by a strictly increasing and strictly Schur-convex function $g$.

The simplest aggregation functions commonly used for the multiple criteria problem (1) are defined as the sum of outcomes,

$g(\mathbf{y})=\sum_{i=1}^{m} y_{i}$

or the worst outcome,

$g(\mathbf{y})=\max _{i=1, \ldots, m} y_{i}$.

The sum (22) is a strictly increasing function while the maximum (23) is only nondecreasing. Therefore, the aggregation (17) using the sum of outcomes always generates a Pareto-optimal solution while the minimization of the worst outcome may need some additional refinement [18]. Both the functions are symmetric and satisfy the requirement (21), although they do not satisfy the equitability requirement (20). Hence, they are Schur-convex but not strictly Schur-convex. Therefore, the corresponding aggregation (17), in the general case, may generate solutions which are not equitably efficient. To generate equitably efficient solutions, some convexification is required.

For any strictly convex, increasing function $s: R \rightarrow R$, the function

$$
g(\mathbf{y})=\sum_{i=1}^{m} s\left(y_{i}\right)
$$

is a strictly monotonic and strictly Schur-convex function [13]. This defines a family of the equitable aggregations according to the following corollary [6].

Corollary 2. For any strictly convex, increasing function $s: R \rightarrow R$, the optimal solution of the problem 
$\min \left\{\sum_{i=1}^{m} s\left(f_{i}(\mathbf{x})\right): \mathbf{x} \in Q\right\}$

is an equitably efficient solution of the multiple criteria problem (1).

Various convex functions $s$ can be used to define the aggregation (25). In the case of the outcomes restricted to positive values, any $p$-power $\alpha^{p}$ is a strictly positive and convex function for $p>1$. This justifies the well known Hölder $L_{p}$ norms

$\|\mathbf{y}\|_{p}=\left(\sum_{i=1}^{m}\left|y_{i}\right|^{p}\right)^{1 / p}$

as a source of equitable aggregations. Specifically, the minimization of $\|\mathbf{y}\|_{p}$ is equivalent to the minimization of $\|\mathbf{y}\|_{p}^{p}=\sum_{i=1}^{m}\left|y_{i}\right|^{p}$ which is a strictly increasing and strictly Schur-convex function for $1<p<\infty$ and positive arguments $y_{i}$.

Note that the sum of outcomes (22) and the worst outcome (23) also represent the $L_{p}$ norms for $p=1$ and $p=\infty$, respectively. Hence, they are limiting cases of the strictly Schur-convex aggregations related to $1<p<\infty$. As the limiting cases they satisfy the corresponding weak requirements. Actually, $L_{1}$ is strictly monotonic but only weakly Schur-convex while $L_{\infty}$ is weakly monotonic and weakly Schur-convex. On the other hand, these two norms can be directly extended to piecewise linear aggregation functions (22) and (23) which are well defined for any outcome values (including negative ones) preserving their properties of (weak) monotonicity and Schur-convexity. The strictly Schur-convex $L_{p}$ norms are nonlinear and there is no direct way to extend them for negative outcomes preserving their strict monotonicity properties. For general (positive and negative) outcomes, one may consider aggregations

$S_{p}(\mathbf{y})=\left(\sum_{i=1}^{m}\left(\max \left\{0, y_{i}\right\}\right)^{p}\right)^{1 / p}$

which are monotonic and Schur-convex but not strictly. Hence, we have two limiting piecewise linear limiting aggregations and a family of nonlinear functions to build intermediate preferences. We will argue further that the space between the piecewise linear aggregation functions (22) and (23) can be filled out with a family of piecewise linear functions which are well defined for any outcome values (including negative ones) maintaining their properties of strict monotonicity and Schur-convexity.

Another way to build equitable aggregations is based on the use of the cumulative ordered outcomes $\bar{\theta}_{i}(\mathbf{y})$. Note that Corollary 1 allows one to generate equitably efficient solutions of (1) as efficient solutions of problem (12). The aggregation minimizing the sum of outcomes, corresponds to minimization of the last ( $m$ th) objective in problem (12). Similar, the minimax scalarization corresponds to minimization of the first objective in (12). In general, one may consider increasing functions of cumulative ordered outcomes $\bar{\theta}_{i}(\mathbf{y})$. In particular, for the weighted sum one gets

$\sum_{i=1}^{m} w_{i} \bar{\theta}_{i}(\mathbf{y})$.

Note that, due to the definition of map $\bar{\theta}$ with (9), the above function can be expressed in the form with weights $v_{i}=\sum_{j=i}^{m} w_{j}(i=1, \ldots, m)$ allocated to coordinates of the ordered outcome vector. Such an approach to aggregation of outcomes was introduced by Yager [27] as the so-called ordered weighted averaging (OWA). When applying OWA to problem (1) we get

$\min \left\{\sum_{i=1}^{m} v_{i} \theta_{i}(\mathbf{f}(\mathbf{x})): \mathbf{x} \in Q\right\}$.

The OWA aggregation is obviously a piecewise linear function since it remains linear within every area of the fixed order of arguments.

Theorem 2. If weights $v_{i}$ are strictly decreasing and positive, i.e. $v_{1}>v_{2}>\cdots>v_{m-1}>v_{m}>0$, then each optimal solution of the OWA problem (29) is an equitably efficient solution of (1).

While equal weights define the linear aggregation, several decreasing sequences of weights lead to various strictly Schur-convex and strictly monotonic aggregation functions. Thus, the monotonic OWA aggregations provide a family of piecewise linear aggregations filling out the space 
between the piecewise linear aggregation functions (22) and (23) as shown in Fig. 2. Actually, formulas (28) and (13) allow us to formulate any monotonic (not necessarily strictly) OWA problem (29) as the following extension of the original multiple criteria problem by linear constraints:

$\min \sum_{k=1}^{m} w_{k} z_{k}$

subject to $\mathbf{x} \in Q$,

$$
\begin{aligned}
& z_{k}=k t_{k}+\sum_{i=1}^{m} d_{i k}^{+} \quad \text { for } k=1, \ldots, m, \\
& t_{k}+d_{i k}^{+} \geqslant f_{i}(\mathbf{x}), \quad d_{i k}^{+} \geqslant 0 \quad \text { for } i, k=1, \ldots, m,
\end{aligned}
$$

where $w_{m}=v_{m}$ and $w_{k}=v_{k}-v_{k+1}$ for $k=$ $1, \ldots, m-1$ (compare [20] for further details).

When differences among weights tend to infinity, the OWA aggregation approximates the leximin ranking of the ordered outcome vectors $[1-3,15,28]$. That means, as the limiting case of the OWA problem (29), we get the lexicographic problem

$\operatorname{lexmin}\{\Theta(\mathbf{f}(\mathbf{x})): \mathbf{x} \in Q\}$

which represents the lexicographic minimax approach (called also the nucleolar approach [11]) to the original multiple criteria problem (1). Problem (33) is a regularization of the standard minimax scalarization (23), but in the former, in addition to the largest outcome, we minimize also the second largest outcome (provided that the largest one remains as small as possible), minimize the third largest (provided that the two largest remain as small as possible), and so on. Due to (9), problem (33) is equivalent to the problem

$\operatorname{lexmin}\{\bar{\Theta}(\mathbf{f}(\mathbf{x})): \mathbf{x} \in Q\}$.

As the lexicographic optimization generates efficient solutions, due to Corollary 1, the optimal solution of the lexicographic minimax problem (33) is an equitably efficient solution of the multiple criteria problem (1). In other words, the minimax aggregation (23) can be lexicographically regularized to guarantee that the corresponding preference relation meets both the strict monotonicity and the principle of transfer (strict Schurconvexity) requirements.

The lexicographic minimax solution can be considered in some sense the "most equitable solution". One may wish to look for a strictly monotonic and Schur-convex regularization of the minisum aggregation (22) thereby generating the "least equitable solution". This can be achieved by applying reverse lexicographic minimization to the problem (12), i.e. solving the lexicographic problem

$\operatorname{lexmin}\left\{\left(\bar{\theta}_{m}(\mathbf{f}(\mathbf{x})), \bar{\theta}_{m-1}(\mathbf{f}(\mathbf{x})), \ldots, \bar{\theta}_{1}(\mathbf{f}(\mathbf{x}))\right): \mathbf{x} \in Q\right\}$,

where first $\bar{\theta}_{m}(\mathbf{f}(\mathbf{x}))$ is minimized, next $\bar{\theta}_{m-1}(\mathbf{f}(\mathbf{x}))$ and so on. Note, that in the lexicographic optimization problem dividing objectives by constants does not affect the solution and $\bar{\theta}_{i}(\mathbf{y}) / i$ represents the mean of $i$ largest coefficients in the outcome vector $\mathbf{y}$. Therefore, we refer to problem (35) as the

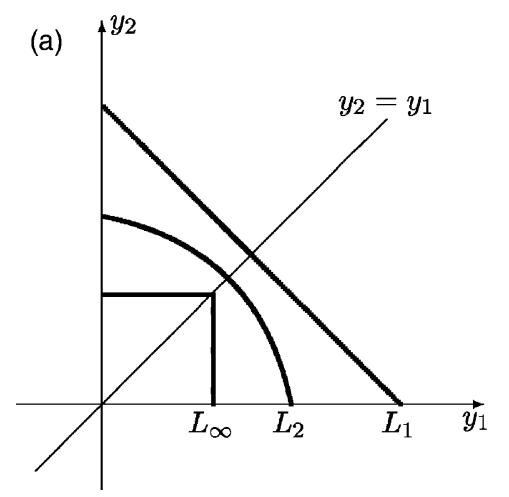

(b)

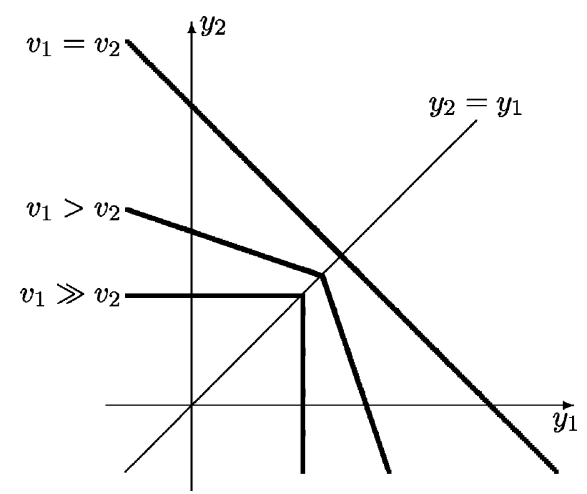

Fig. 2. Isoline contours for equitable aggregations: (a) $L_{p}$ norms, and (b) OWA aggregations. 
lexicographic mean problem. It follows from Corollary 1 that the optimal solution of the lexicographic mean problem (35) is an equitably efficient solution of the multiple criteria problem (1).

\section{Applications}

Recently, there have appeared a number of papers dealing with issues of equity and even some papers which consider multiple criteria equity models $([4,9,10,16,17]$ and references therein). In some of these papers, the solutions presented are equitably efficient, but the authors do not acknowledge this fact (cf. references in [10]), while in other papers, computed Pareto-optimal solutions are criticized for their lack of equity [4,9] and the authors replace the search for an equitably efficient solution by minimization of some inequality measures or even formally abandon the entire multiple criteria model. Since the Pareto-optimal set is very large in some models, it is quite possible to compute efficient solutions which are very far from the equitable efficient solutions. Thus, even though some quite appealing equitably efficient solutions may exist, they may be ignored in favor of solutions which are less appealing, and less justified by mathematical principles. In Section 4.1 we show the real-life case of the budget redistribution [4] may be effectively solved with the use of equitable aggregations.

On the other hand, several multiple criteria optimization methods build the individual achievement functions which measure actual achievement of each outcome with respect to the corresponding preference parameters. Thus all the original outcomes are transformed into a uniform scale of individual achievements allowing one to use some impartial aggregation techniques. This applies, in particular, to the wide family of the reference point method and goal programming approaches. For these approaches, equity among the individual achievements has been raised as an important issue (cf. [18] and references therein). In Section 4.2 we will show that every efficient solution to any multiple criteria problem can be found by equitable optimization of appropriate individual achievement functions. We especially focus on the reference point methods taking advantages of this relation.

\subsection{Equitable preferences}

Budgets, in the administration of organizations, have become increasingly dynamic. Cuts in budgets get increased publicity, and equity is sought in how to apply these cuts. However, budgets may also reflect increases, which should also be applied in a fair (equitable) manner. In this section we examine the case treated by Fandel and Gal [4], and we show that it may be solved in quite a satisfying way by means of equitably efficient solutions. Fifteen state universities in North RhineWestphalia together with the German Ministry of Science and Research participated in the redistribution of a part of the budget for teaching and research. The authors reported on how the redistribution problem has been treated by methods of Operations Research and how the final solution was reached in a process of negotiations between the Ministry and participating universities. The decision makers agreed on a set of 5 measures of university performance. The measures were calculated for each of the 15 universities, resulting in constants that were combined using attribute shares $g_{1}, g_{2}, \ldots, g_{5}$. These shares were treated as decision variables in the subsequent models of the decision problem.

The universities participating in the redistribution requested that the resulting distribution should be as close as possible to the original distribution. Thus, the problem was modeled using 15 criteria $\left|z_{i}\right|$, which represent the absolute deviation of the new budget from the original budget of each university. These criteria are obviously impartial and equitable. Actually, several $L_{p}$ norms have been applied while looking for a solution [4]. We present these solutions in the first three rows of Table 1. None of the solutions have been accepted by the Ministry. Finally, the optimization was abandoned and after the negotiation process a solution (reported in the table as 'chosen') has been selected, that satisfied additional requirements of the decision makers, which will be discussed below. 
Table 1

Solutions found by simple OWA models

\begin{tabular}{lllllllll}
\hline Model & $g_{1}$ & $g_{2}$ & $g_{3}$ & $g_{4}$ & $g_{5}$ & $\max _{i}\left|z_{i}\right|$ & $\sum_{i}\left|z_{i}\right|$ & $\sum_{i}\left|z_{i}\right|^{2}$ \\
\hline$L_{1}$ & 0.0000 & 0.2614 & 0.5120 & 0.1746 & 0.0520 & 1.30 & 6.39 & 4.74 \\
$L_{2}$ & 0.5030 & 0.2647 & 0.4648 & 0.1720 & 0.0482 & 1.19 & 6.86 & 4.52 \\
$L_{\infty}$ & 0.1546 & 0.1348 & 0.5051 & 0.2045 & 0.0000 & 0.92 & 7.90 & 5.19 \\
Chosen & 0.20 & 0.20 & 0.35 & 0.20 & 0.05 & 1.37 & 7.90 & 6.25 \\
Minimax & 0.15 & 0.15 & 0.50 & 0.20 & 0.00 & 0.95 & 7.83 & 5.44 \\
Minisum & 0.00 & 0.30 & 0.45 & 0.20 & 0.05 & 1.22 & 6.61 & 4.71 \\
OWA-1 & 0.05 & 0.30 & 0.45 & 0.15 & 0.05 & 1.26 & 6.97 & 4.67 \\
OWA-2 & 0.00 & 0.30 & 0.50 & 0.15 & 0.05 & 1.39 & 6.82 & 4.80 \\
OWA-3 & 0.10 & 0.25 & 0.45 & 0.15 & 0.05 & 1.12 & 7.18 & 4.62 \\
Optimal & 0.10 & 0.30 & 0.35 & 0.15 & 0.10 & 1.04 & 7.26 & 5.01 \\
\hline
\end{tabular}

We wish to use the case to demonstrate that a consequent application of equitable optimization, particularly the OWA aggregation, could directly lead to acceptable results. For this purpose we have computed several OWA aggregations of the criteria. For comparability with the 'chosen' solution, we have limited the decision variables $g_{k}$ to the discrete grid of values: $g_{k} \in\{0.0,0.05, \ldots$, $0.95,1.0\}$. Since the equitable OWA aggregation as implementable by LP (30)-(32) is capable to accommodate additional integer (discrete) constraints, this is easily accomplished. Table 1 reports results for some simple OWA models. We have started with the minimax and minisum approaches as the limiting OWA models defined with weights $w_{i}$ given by the sequences $1,0, \ldots, 0$ and $0, \ldots, 0,1$, respectively. Next we consider models defined by $w_{i}=1$ for all $i$ (OWA-1), $w_{i}=i$ (OWA$2)$, and $w_{i}=16-i$ (OWA-3). Note that we define the OWA models by positive weights $w_{i}$ as in (28) and LP formulation (30)-(32). Hence, in terms of (29), OWA-1 represents strictly monotonic weights $v_{i}$ decreasing with constant step 1. In OWA-2 weights $v_{i}$ decrease faster for large $i$ which makes the corresponding solution closer to the minisum result while opposite weights $v_{i}$ decreasing faster for small numbers $i$ in OWA-3 makes the solution closer to the minimax model.

While looking for the distribution scheme the decision maker wanted to avoid too large attribute shares $g_{k}$ allocated to a single decision factor (evaluation criterion) as well as zero shares excluding some factors from the decision process [4].
We have enforced these additional requirements by restricting the attribute shares $g_{k}$ to the interval: $0.0<g_{k}<0.4$. It turns out that all our OWA models result then in the same equitably efficient solution presented as 'optimal' in Table 1. One can easily notice that the solution is much better than 'chosen' in terms of all the $L_{p}$ measures. Actually, the 'optimal' solution equitably dominates the 'chosen' one, as clearly shown with the corresponding absolute Lorenz curves in Fig. 3 (note that normalizing factor $1 / 15$ is ignored for both the axes as we start the curves from the first ordered criterion thus depicting the minimax values). Moreover, the solution 'optimal' meets also other additional requirements and restrictions mentioned in [4]. We do not want to question a

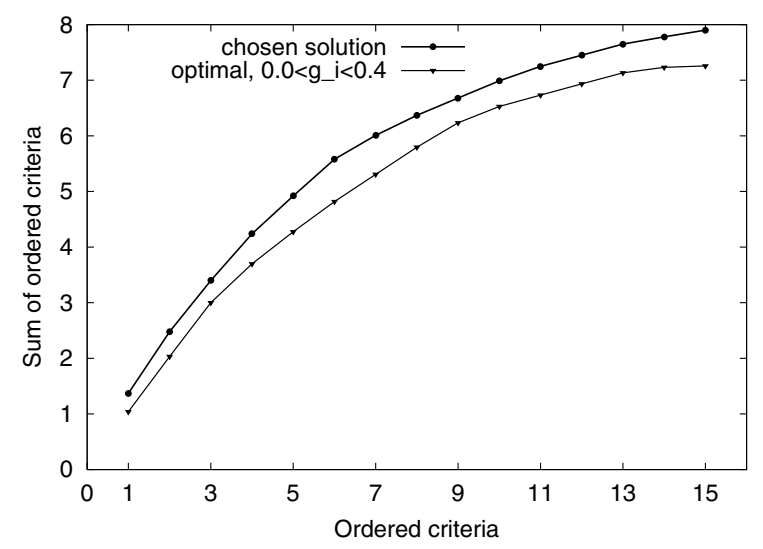

Fig. 3. Absolute Lorenz curves: solution 'chosen' dominated by 'optimal'. 
solution accepted for that application. We rather wish to show that there exist methodological tools to model various equitable preferences.

To demonstrate a wider gamut of the equitable preferences supported by OWA modeling we have built and solved several more complex models. Table 2 presents results for four such models. All of them were analyzed on the basic decision problem without any upper bound on $g_{k}$. Model OWA-4 is defined by weights $w_{i}=i^{2}$ which makes it closer to the minisum model than OWA-2. It turns out that the model generates exactly the same solution as the standard minisum approach. This justifies the minisum solution from Table 1 as the equitably efficient one. Similarly, one may consider weights $w_{i}=(16-i)^{2}$ or $w_{i}=(16-i)^{3}$ to strengthen the OWA-3 model. The latter, denoted as OWA-5, turns out to be closer to the minimax model than OWA-3 but not very much closer. In order to enforce solutions closer to the minimax model one may directly increase the weight corresponding to this criterion. The OWA- 6 model defined by weights $w_{1}=2000$ and $w_{i}=i^{2}$ for $i=2, \ldots, 15$ has additional stress on the minimax criterion as it represents a linear combination of the minimax criterion $\times 1999$ plus the OWA- 5 criterion. Similar, the OWA-7 model defined by weights $w_{1}=500$ and $w_{i}=1$ for $i=2, \ldots, 15$ can be interpreted as a linear combination of the minimax criterion $\times 499$ plus the OWA-1 criterion. The latter turns out to be almost optimal with respect to the minimax criterion $\left(L_{\infty}\right.$ norm) and generating better values of the $L_{1}$ and $L_{2}$ norms than the original minimax solution, while the former generates even better values of the $L_{1}$ and $L_{2}$ norms but worsening the minimax criterion. Note that in terms of the three $L_{p}$ norms, we consider, the OWA-2 and OWA-5 solutions are worse than the results of OWA-6. Nevertheless, both OWA-2

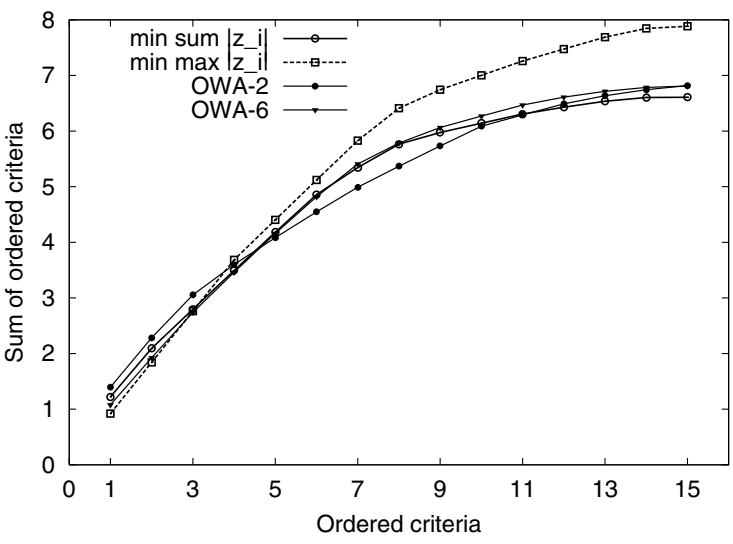

Fig. 4. Absolute Lorenz curves for the sample problem.

and OWA-5 are equitably efficient. The entire absolute Lorenz curves are important to define the equitable dominance and Fig. 4 depicts various plots of the curves showing that the OWA-2 curve is the best for intermediate numbers of criteria.

\subsection{Nonequitable preferences}

Standard multiple criteria optimization problems with a general preference structure essentially assume the criteria to be incomparable, i.e. having no basis of comparison. Therefore, they cannot be directly considered as equitable. Nevertheless, typical multiple criteria optimization methods aggregate the outcomes with various scalarizing functions [14,25]. We argue that most scalarizing functions can be viewed as two-stage transformations of the original outcomes. At the first stage, the individual achievement functions are built which measure actual achievement of each outcome with respect to the corresponding preference parameters. Thus, all the outcomes are transformed into a uniform scale of individual

Table 2

Solutions found by complex OWA models

\begin{tabular}{lllllllll}
\hline Model & $g_{1}$ & $g_{2}$ & $g_{3}$ & $g_{4}$ & $g_{5}$ & $\max _{i}\left|z_{i}\right|$ & $\sum_{i}\left|z_{i}\right|$ & $\sum_{i}\left|z_{i}\right|^{2}$ \\
\hline OWA-4 & 0.00 & 0.30 & 0.45 & 0.20 & 0.05 & 1.22 & 6.61 & 4.71 \\
OWA-5 & 0.10 & 0.20 & 0.50 & 0.20 & 0.00 & 1.09 & 7.49 & 4.91 \\
OWA-6 & 0.05 & 0.25 & 0.45 & 0.20 & 0.05 & 1.08 & 6.81 & 4.67 \\
OWA-7 & 0.10 & 0.20 & 0.45 & 0.20 & 0.05 & 0.96 & 7.06 & 5.02 \\
\hline
\end{tabular}


achievements. At the second stage the individual achievements are impartially aggregated in order to select a better distribution of achievements. This allows one to apply the equitable techniques to aggregate the individual achievements. Such an approach is justified by the following assertions.

Theorem 3. If $\overline{\mathbf{x}} \in Q$ is a Pareto-optimal solution to multiple criteria problem (1) and $s_{i}: R \rightarrow R$ are strictly increasing scaling functions satisfying the requirement

$s_{1}\left(f_{1}(\overline{\mathbf{x}})\right)=s_{2}\left(f_{2}(\overline{\mathbf{x}})\right)=\cdots=s_{m}\left(f_{m}(\overline{\mathbf{x}})\right)=\tau$

with some arbitrary real value $\tau$, then $\overline{\mathbf{x}}$ is an equitably efficient solution to scaled problem

$\min \left\{\left(s_{1}\left(f_{1}(\mathbf{x})\right), \ldots, s_{m}\left(f_{m}(\mathbf{x})\right)\right): \mathbf{x} \in Q\right\}$.

Proof. Suppose that $\overline{\mathbf{x}} \in Q$, Pareto-optimal to multiple criteria problem (1) is not equitably efficient to (37). Then, there exists a feasible $\mathbf{x} \in Q$ such that $\mathbf{y}^{s}=\left(s_{1}\left(f_{1}(\mathbf{x})\right), \ldots, s_{m}\left(f_{m}(\mathbf{x})\right)\right)$ equitably dominates $\quad \overline{\mathbf{y}}^{s}=\left(s_{1}\left(f_{1}(\overline{\mathbf{x}})\right), \ldots, s_{m}\left(f_{m}(\overline{\mathbf{x}})\right)\right)$. This means that $\bar{\theta}_{i}\left(\mathbf{y}^{s}\right) \leqslant \bar{\theta}_{i}\left(\overline{\mathbf{y}}^{s}\right)$ for $i=1,2, \ldots, m$ with at least one inequality strict. However, due to (36), $\bar{\theta}_{i}\left(\overline{\mathbf{y}}^{s}\right)=\tau$ for all $i=1,2, \ldots, m$. Hence,

$\sum_{i=1}^{k} s_{i}\left(f_{i}(\mathbf{x})\right) \leqslant k \tau=\sum_{i=1}^{k} s_{i}\left(f_{i}(\overline{\mathbf{x}})\right)$ for $k=1,2, \ldots, m$

and, due to strictly increasing functions $s_{i}$, one gets $f_{i}(\mathbf{x}) \leqslant f_{i}(\overline{\mathbf{x}})$ for $i=1,2, \ldots, m$ with at least one inequality strict, which contradicts the Paretooptimality of $\overline{\mathbf{x}}$.

Corollary 3. For any $\overline{\mathbf{x}} \in Q$ Pareto-optimal solution to multiple criteria problem (1) there exist strictly increasing scaling functions such that $\overline{\mathbf{x}}$ is an equitably efficient solution to scaled problem (37).

Note that as a sample scaling functions satisfying the requirement (36) one may consider $s_{i}\left(y_{i}\right)=y_{i}-f_{i}(\overline{\mathbf{x}})$ or $s_{i}\left(y_{i}\right)=\left(y_{i}-b_{i}\right) /\left(f_{i}(\overline{\mathbf{x}})-b_{i}\right)$ where $b_{i}<f_{i}(\overline{\mathbf{x}})$ is an arbitrary parameter. Thus there exist linear scaling functions meeting the requirements of Theorem 3. Actually, this type of scaling function is used in the reference point method (RPM) to form the so-called individual (or partial) achievement functions.
Recall that the idea of RPM is to produce several efficient solutions according to the DM preferences specified interactively in terms of reference (aspiration) levels. Depending on the specified reference levels a scalarizing achievement function is built which, when optimized, generates an efficient solution to the problem. The scalarizing achievement function may be directly interpreted as expressing utility to be maximized. However, to keep the discussion consistent with the minimization models we will assume that the scalarizing achievement function is minimized (thus representing dis-utility). The generic scalarizing achievement function takes then the following form:

$\max _{1 \leqslant i \leqslant m}\left\{s_{i}\left(f_{i}(\mathbf{x})\right)\right\}+\varepsilon \sum_{i=1}^{m} s_{i}\left(f_{i}(\mathbf{x})\right)$,

where $\varepsilon$ is an arbitrary small positive number and $s_{i}\left(y_{i}\right)=s_{i}\left(y_{i}, b_{i}\right)$, for $i=1,2, \ldots, m$, are the individual achievement functions measuring actual achievement of the $i$ th outcome with respect to the corresponding reference levels $b_{i}$.

The standard RPM methodology [26] assumes the parameter $\varepsilon$ in formula (38) to be arbitrarily small. Thus, when accepting the loss of a direct utility interpretation, one may consider a limiting case with $\varepsilon \rightarrow 0_{+}$which results in lexicographic order applied to two separate terms of function (38). Therefore, RPM may be also considered as the following lexicographic problem ([18] and references therein):

$\operatorname{lexmin}\left\{\left[\max _{1 \leqslant i \leqslant m}\left\{s_{i}\left(f_{i}(\mathbf{x})\right)\right\}, \sum_{i=1}^{m} s_{i}\left(f_{i}(\mathbf{x})\right)\right]: \mathbf{x} \in Q\right\}$.

The advantage of the above lexicographic model is that it allows us to generate all efficient solutions whereas only properly efficient solutions can be obtained with the minimization of (38).

Various functions $s_{i}$ provide a wide modeling environment for measuring individual achievements. For the sake of computational robustness, the piecewise linear functions $s_{i}$ are usually employed. In the simplest models, they take a form of two segment piecewise linear functions 
$s_{i}\left(y_{i}\right)= \begin{cases}\lambda_{i}^{+}\left(y_{i}-b_{i}\right) & \text { for } y_{i} \geqslant b_{i}, \\ \lambda_{i}^{-}\left(y_{i}-b_{i}\right) & \text { for } y_{i}<b_{i},\end{cases}$

where $\lambda_{i}^{+}$and $\lambda_{i}^{-}$are positive scaling factors corresponding to underachievements and overachievements, respectively, for the $i$ th outcome. It is usually assumed that $\lambda_{i}^{+}$is much larger than $\lambda_{i}^{-}$. However, even linear functions

$s_{i}\left(y_{i}\right)=\lambda_{i}\left(y_{i}-b_{i}\right)$

with positive scaling factors $\lambda_{i}$ represent simplified (but still valid) individual achievement functions. Real-life applications of the RPM methodology usually deal with more complex individual achievement functions defined with more than one reference point [26] which enriches the preference models and simplifies the interactive analysis. In particular, the so-called aspiration/reservation models [8] are used which take advantages of two reference levels. In addition to the main (aspiration) levels $b_{i}$ they employs also reservation levels $r_{i}\left(r_{i}>b_{i}\right)$, so that the DM can specify desired as well as required values for given outcomes. The piecewise linear individual achievement function may be defined than as follows [19]:

$s_{i}\left(y_{i}\right)= \begin{cases}\gamma \frac{y_{i}-r_{i}}{r_{i}-b_{i}}+1 & \text { for } y_{i}>r_{i}, \\ \frac{y_{i}-b_{i}}{r_{i}-b_{i}} & \text { for } b_{i}<y_{i} \leqslant r_{i}, \\ \beta \frac{y_{i}-b_{i}}{r_{i}-b_{i}} & \text { for } y_{i} \leqslant b_{i},\end{cases}$

where $\beta$ and $\gamma$ are arbitrarily defined parameters satisfying $0<\beta<1<\gamma$. Independently from the specific form of the individual achievement functions, their main properties remain. Namely, for any reference value $b_{i}$, function $s_{i}\left(y_{i}\right)$ must be strictly increasing with respect to $y_{i}$ (the $i$ th outcome) and it has to take a common value (usually 0 ) for $y_{i}=b_{i}$. Hence, the following assertion may be derived from Theorem 3 .

Corollary 4. For any RPM individual achievement functions $s_{i}\left(y_{i}\right)$, if $\overline{\mathbf{x}} \in Q$ is a Pareto-optimal solution to multiple criteria problem (1), then $\overline{\mathbf{x}}$ is also an equitably efficient solution to the multiple achievement optimization problem

$$
\begin{gathered}
\min \left\{\left(a_{1}, a_{2}, \ldots, a_{m}\right): a_{i}=s_{i}\left(f_{i}(\mathbf{x})\right),\right. \\
i=1, \ldots, m ; \mathbf{x} \in Q\}
\end{gathered}
$$

with $b_{i}=f_{i}(\overline{\mathbf{x}})$ for $i=1,2, \ldots, m$.

In other words, the RPM (individual) achievement functions form a new uniform multiple criteria problem where the analysis can be focused on equitably efficient solutions. Actually, the standard RPM model with the analytic scalarizing achievement function (38) can be expressed as the following OWA model:

$$
\begin{gathered}
\min \left\{\left[(1+\varepsilon) \theta_{1}(\mathbf{a})+\varepsilon \sum_{i=2}^{m} \theta_{i}(\mathbf{a})\right]: a_{i}=s_{i}\left(f_{i}(\mathbf{x})\right),\right. \\
i=1, \ldots, m ; \mathbf{x} \in Q\} .
\end{gathered}
$$

Hence, the standard RPM model exactly represents the analytic (utility) form of the equitable OWA aggregation (29) with strictly decreasing weights in the case of $m=2\left(v_{1}=1+\varepsilon>v_{2}=\varepsilon\right)$. For $m>2$ it abandons the differences in weighting of the second largest achievement, the third largest one etc. $\left(v_{2}=v_{3}=\cdots=v_{m}=\varepsilon\right)$. This results in an approximation to the equitable optimization defined by the OWA aggregation satisfying only weak Schur-convexity property (21).

Similarly, the lexicographic RPM model (39) can be expressed as the following problem:

$$
\begin{gathered}
\operatorname{lexmin}\left\{\left[\theta_{1}(\mathbf{a}), \sum_{i=2}^{m} \theta_{i}(\mathbf{a})\right]: a_{i}=s_{i}\left(f_{i}(\mathbf{x})\right),\right. \\
i=1, \ldots, m ; \mathbf{x} \in Q\}
\end{gathered}
$$

thus, in the case of two criteria $(m=2)$, representing exactly the lexicographic minimax (33) approach to the multiple achievement optimization problem (43). For larger number of criteria $(m>2)$ model (39) only approximates the lexicographic minimax (33) as all the lower priority objective terms are aggregated at the second priority level. Hence, the lexicographic RPM model (39) fulfills the principle of transfers only in the case of an improvement of the worst individual achievement.

One may consider the lexicographic minimax problem (33) applied to the multiple criteria achievement problem (43) as a basis for a corresponding nucleolar RPM model 


$$
\begin{gathered}
\operatorname{lexmin}\left\{\left[\theta_{1}(\mathbf{a}), \theta_{2}(\mathbf{a}), \ldots, \theta_{m}(\mathbf{a})\right]: a_{i}=s_{i}\left(f_{i}(\mathbf{x})\right),\right. \\
i=1, \ldots, m ; \mathbf{x} \in Q\} .
\end{gathered}
$$

The nucleolar RPM represents a true equitable optimization of the individual achievements. Actually it implements the Rawls principle of justice [22]. In order to illustrate modeling advantages of the nucleolar RPM let us consider two possible achievement vectors: $\mathbf{a}^{\prime}=(10,0,0,-5)$ thus representing the solution leaving only one aspiration level not reached and $\mathbf{a}^{\prime \prime}=(10,10,10,-30)$ with three aspiration levels not satisfied. One may easily notice that the first one will be selected by the nucleolar RPM while a" would be chosen by the standard RPM. Hence, the equitable optimization implemented within the nucleolar RPM results in much better modeling of the aspiration levels concept.

The nucleolar RPM model (44) can be expressed in terms of the lexicographic minimization of the quantities $\bar{\theta}_{i}(\mathbf{a})$ :

$$
\begin{aligned}
\operatorname{lexmin} & \left\{\left[\bar{\theta}_{1}(\mathbf{a}), \bar{\theta}_{2}(\mathbf{a}), \ldots, \bar{\theta}_{m}(\mathbf{a})\right]: a_{i}=s_{i}\left(f_{i}(\mathbf{x})\right), i\right. \\
& =1, \ldots, m ; \mathbf{x} \in Q\}
\end{aligned}
$$

and thereby it is quite easily implementable. Exactly, following the results from Section 2, it can be considered as a standard lexicographic optimization:

$\operatorname{lexmin}\left[z_{1}, z_{2}, \ldots, z_{m}\right]$

subject to $\mathbf{x} \in Q$,

$$
\begin{aligned}
& z_{k}=k t_{k}+\sum_{i=1}^{m} d_{i k}^{+} \quad \text { for } k=1, \ldots, m, \\
& t_{k}+d_{i k}^{+} \geqslant s_{i}\left(f_{i}(\mathbf{x})\right), \quad d_{i k}^{+} \geqslant 0 \quad \text { for } i, k=1, \ldots, m .
\end{aligned}
$$

In the case of convex piecewise linear individual achievement functions (as typically used in the RPM approaches), the resulting formulation (45)(47) extends the original constraints with linear inequalities. Thus, the method can be effectively applied to various multiple criteria problems including the discrete ones.

\section{Concluding remarks}

The concept of equitably efficient solutions is a specific refinement of Pareto-optimality. Hence, equitable multiple criteria techniques focus on some selection of Pareto-optimal solutions. It turns out, however, that there are many applications in which the criteria are uniform in the sense of the scale used and their values are directly comparable. Moreover, the criteria are considered impartially, which makes the distribution of outcomes more important than the assignment of several outcomes to the specific criteria. Note that having two possible solutions generating outcome vectors $(5,0,5)$ and $(0,3,0)$, respectively, we recognize both the solutions as Pareto-optimal. However, the first solution generates two outcomes equal to 5 and one outcome equal to 0 , whereas the second solution generates one outcome equal to 3 and two outcomes equal to 0 . Thus, the second outcome vector is clearly better in terms of distribution of outcomes and the concept of equitable efficiency allows us distinguish these two solutions.

Typical solution concepts for multiple criteria problems are defined by aggregations of the original criteria. In order to guarantee the consistency of the aggregated problem with minimization of all individual objective functions in the original multiple criteria problem, the aggregation function must be strictly increasing with respect to every coordinate. Every optimal solution of the aggregated optimization is then a Pareto-optimal solution to the original multiple criteria problem. In order to generate equitably efficient solutions, the aggregation functions must be also symmetric and maintain some convexity properties (be Schur-convex). In the case of the outcomes restricted to positive values the norms can be used as aggregation functions. We have demonstrated in this paper that much better results can be achieved with the ordered weighted averaging (OWA) aggregations. The OWA aggregations provide a family of piecewise linear functions allowing to model various equitable preferences. They can be easily implemented as extensions of the original problem by linear constraints. 
There are many applications in which the criteria express ideas of allocation of resources and try to achieve some equitable allocation of resources. We have examined in detail the case [4] of budget redistribution, and we have shown that it may be solved in a quite satisfying way by means of equitable optimization. Particularly, the use of the OWA aggregation could directly lead to acceptable results, while originally applied norms resulted in unsatisfactory solutions and required additional negotiation process to select the final solution.

Moreover, equitable optimization techniques can also be applied to select efficient solutions in general multiple criteria optimization. Indeed, in approaches which seek to scalarize the multiple criteria, some effort is always placed to replace the original objective functions with some individual achievements which are combined to form a final scalar objective function to be optimized. This is done, for example, in the reference point method, which we have analyzed in detail. We have shown that every efficient solution of a multiple criteria optimization problem can be identified by the optimization of an equitable aggregation applied to appropriately defined individual achievements and we have introduced an equitable model of the reference point method.

Although the examples considered to date have come from linear and integer multiple criteria optimization, the theory included in the paper elegantly covers also the area of nonlinear multiple criteria optimization, which has great potential for impact in financial analysis and engineering design.

\section{References}

[1] F.A. Behringer, Linear multiobjective maxmin optimization and some Pareto and lexmaxmin extensions, OR Spektrum 8 (1986) 25-32.

[2] D. Dubois, Ph. Fortemps, M. Pirlot, H. Prade, Leximin optimality and fuzzy set-theoretic operations, European Journal of Operational Research 130 (2001) 20-28.

[3] M. Ehrgott, Discrete decision problems, multiple criteria optimization classes and lexicographic max-ordering, in: T.J. Stewart, R.C. van den Honert (Eds.), Trends in Multicriteria Decision Making, Springer, Berlin, 1998, pp. $31-44$.

[4] G. Fandel, T. Gal, Redistribution of funds for teaching and research among universities: The case of North Rhine-
Westphalia, European Journal of Operational Research 130 (2001) 111-120.

[5] J. Kalcsics, S. Nickel, J. Puerto, A. Tamir, Algorithmic results for ordered median problems, Operations Research Letters 30 (2002) 149-158.

[6] M.M. Kostreva, W. Ogryczak, Linear optimization with multiple equitable criteria, RAIRO Operations Research 33 (1999) 275-297.

[7] M.M. Kostreva, W. Ogryczak, Equitable approaches to location problems, in: J.-C. Thill (Ed.), Spatial Multicriteria Decision Making and Analysis-A Geographic Information Sciences Approach, Ashgate, Brookfield, 1999, pp. 103-126.

[8] A. Lewandowski, A.P. Wierzbicki (Eds.), Aspiration Based Decision Support Systems-Theory, Software and Applications, Springer, Berlin, 1989.

[9] M.C. López-de-los-Mozos, J.A. Mesa, The maximum absolute deviation measure in location problems on networks, European Journal of Operational Research 135 (2001) 184-194.

[10] H. Luss, On equitable resource allocation problems: A lexicographic minimax approach, Operations Research 47 (1999) 361-378.

[11] E. Marchi, J.A. Oviedo, Lexicographic optimality in the multiple objective linear programming: The nucleolar solution, European Journal of Operational Research 57 (1992) 355-359.

[12] M.T. Marsh, D.A. Schilling, Equity measurement in facility location analysis: A review and framework, European Journal of Operational Research 74 (1994) 1-17.

[13] A.W. Marshall, I. Olkin, Inequalities: Theory of Majorization and Its Applications, Academic Press, New York, 1979.

[14] K. Miettinen, M.M. Mäkelä, On scalarizing functions in multiobjective optimization, OR Spectrum 24 (2002) 193213.

[15] W. Ogryczak, On the lexicographic minimax approach to location problems, European Journal of Operational Research 100 (1997) 566-585.

[16] W. Ogryczak, Inequality measures and equitable approaches to location problems, European Journal of Operational Research 122 (2000) 374-391.

[17] W. Ogryczak, Multiple criteria linear programming model for portfolio selection, Annals of Operations Research 97 (2000) 143-162.

[18] W. Ogryczak, On goal programming formulations of the reference point method, Journal of the Operational Research Society 52 (2001) 691-698.

[19] W. Ogryczak, K. Studziński, K. Zorychta, DINAS: A computer-assisted analysis system for multiobjective transshipment problems with facility location, Computers and Operations Research 19 (1992) 637-647.

[20] W. Ogryczak, T. Śliwiński, On solving linear programs with the ordered weighted averaging objective, European Journal of Operational Research 148 (2003) 80-91.

[21] W. Ogryczak, A. Tamir, Minimizing the sum of the $k$ largest functions in linear time, Information Processing Letters 85 (2003) 117-122. 
[22] J. Rawls, The Theory of Justice, Harvard University Press, Cambridge, 1971.

[23] R.E. Steuer, Multiple Criteria Optimization-Theory, Computation and Applications, John Wiley and Sons, New York, 1986.

[24] D.J. White, A bibliography on the applications of mathematical programming multiple-objective methods, Journal of the Operational Research Society 41 (1990) 669-691.

[25] A.P. Wierzbicki, A mathematical basis for satisficing decision making, Mathematical Modelling 3 (1982) 391-405.
[26] A.P. Wierzbicki, M. Makowski, J. Wessels (Eds.), Model Based Decision Support Methodology with Environmental Applications, Kluwer, Dordrecht, 2000.

[27] R.R. Yager, On ordered weighted averaging aggregation operators in multicriteria decision making, IEEE Transactions on Systems, Man and Cybernetics 18 (1988) 183-190.

[28] R.R. Yager, On the analytic representation of the Leximin ordering and its application to flexible constraint propagation, European Journal of Operational Research 102 (1997) 176-192. 